\title{
Comportamiento Hidrodinámico y Térmico del Flujo Gas- Sólido en un Reactor de Tubo Vertical (Riser) del Proceso de Craqueo Catalítico (FCC) con dos Salidas Laterales Asimétricas
}

\author{
Ignacio Paz-Paredes ${ }^{(1,2)}$, Juan G. Barbosa-Saldaña ${ }^{(1)}$, Luis A. Moreno-Pacheco ${ }^{(1)}$, Claudia C. Gutiérrez-Torres ${ }^{(1)}$ \\ (1) Instituto Politécnico Nacional. ESIME-SEPI LABINTHAP UPALM, 07738, México D.F.- México \\ (e-mail: jbarbosas@ipn.mx, lamoreno@ipn.mx, cgutierrezt@ipn.mx) \\ (2) Instituto Mexicano del Petróleo. Dirección de Servicios de Ingeniería, 07730 México, D.F. - México \\ (e-mail: jipaz@imp.mx)
}

Recibido Oct. 26, 2015; Aceptado Ene. 15, 2016; Versión final Feb. 16, 2016, Publicado Jun. 2016

\begin{abstract}
Resumen
En este trabajo se realiza la simulación numérica en 2D del comportamiento hidrodinámico y térmico del flujo gas-sólido en un reactor industrial (riser) con dos salidas colocadas asimétricamente, utilizando un modelo de la Dinámica de Fluidos Computacional. El sistema se considera adiabático y el modelo del sistema se resuelve por una aproximación Euleriana transitoria y la Teoría Cinética del Flujo Granular (KTGF, Kinetic Theory for Granular Flow). Como modelo de arrastre se utiliza la minimización multiescala de la energía (EMMS, Energy Minimization Multi-Scale). Las variables que se analizan son la distribución de densidad, la distribución de velocidades, la distribución del flujo másico, y los perfiles de temperatura de la fase sólida, a varias alturas del riser. Los resultados de este trabajo se comparan con los obtenidos anteriormente para una configuración de salidas simétricas. El modelo predice adecuadamente la presencia de tres zonas de concentración y velocidad de sólidos, así como la formación del patrón de flujo anular esperado en el riser.
\end{abstract}

\section{Hydrodynamic and Thermal Behavior of Gas-Solid Flow in an Vertical Pipe Reactor (Riser) of the Fluid Catalytic Cracking Process (FCC) with Two Lateral Asymmetric Outlets}

\begin{abstract}
In this work 2D numerical simulations of gas-solid flow hydrodynamic and thermal behavior in an industrial riser is made using a Computational Fluid Dynamics model. The mixture goes out from the riser through two lateral upper asymmetric outlets. The system is considered to be adiabatic and the system is solved through a transient Eulerian approach and the Kinetic Theory for Granular Flow. The Energy Minimization MultiScale method (EMMS) is used as a drag model. Solid density distribution, velocity distribution, mass flow distribution and temperature profiles measured at several riser heights are analyzed. The results obtained in this work are compared with those previously obtained in a similar system but having two symmetric outlets. The model properly predicts the presence of three solid concentration and velocity zones, as well as the expected core-annular flow pattern in the riser.
\end{abstract}

Keywords: numerical simulation; industrial riser; asymmetric outlets; eulerian approach; EMMS drag model 


\section{INTRODUCCIÓN}

Los lechos fluidizados circulantes (CFB) han sido de amplia aplicación dentro de la industria de transformación y de generación de energía, fundamentalmente debido a su gran capacidad para transferir masa y energía. El riser (tubo vertical) es el equipo principal del CFB donde se lleva a cabo el fenómeno de fluidización, y en su caso las reacciones químicas o de combustión, como en el Proceso de Desintegración Catalítica de Lecho Fluidizado (Proceso FCC) de la industria de la refinación del petróleo, donde un catalizador sólido granulado se pone en contacto con una corriente de vapor de agua de media presión con la velocidad suficiente que provoca que las partículas del sólido se muevan como un fluido (fluidización). Adicionalmente el gasóleo de vacío que es la materia prima del proceso, el cual se convierte a través de reacciones químicas de fragmentación de moléculas, en compuestos más ligeros como gas, gasolina, y aceite cíclico; se atomiza dentro del riser a través de boquillas especialmente diseñadas para este propósito, y al entrar en contacto con el catalizador sólido caliente se vaporiza, y también contribuye a la fluidización del catalizador (Sadeghbeigi, 1995).

Desde hace tres décadas varios investigadores han logrado simular con cierto éxito el comportamiento hidrodinámico del flujo gas-sólidos dentro de un riser (Neri y Gidaspow, 2000; Reddy-Karri y Knowlton, 1999; Pärssinen y Zhu, 2001; Cabezas-Gómez y Milioli, 2003), como primera aproximación utilizando modelos de arrastre homogéneos como los propuestos por Gidaspow (1993), Syamlal-O'Brien (1987), Wen-Yu (1966), entre otros; los cuales no son capaces de predecir la formación de cluters (grupos de partículas) por lo que tampoco predicen el flujo descendente de los sólidos.

Los patrones y perfiles del flujo gas-sólidos que se desarrollan dentro del riser dependen de varios factores tales como las condiciones de operación, las propiedades del medio fluidizante y de los sólidos, la geometría del riser, y la forma en que están dispuestas las entradas y salidas de las corrientes. La simulación numérica de este proceso, tratando de predecir el comportamiento del flujo gas-sólidos, evaluando los perfiles de concentración y velocidad de los sólidos así como el perfil de temperatura, se han venido realizando desde las últimas dos décadas del siglo pasado, fundamentalmente variando la geometría y se ha observado que al variarla se modifican los perfiles de flujo a lo largo de todo el riser (Huilin et al., 2006; Hamzehei y Rahimzadeh, 2010; Chalermsinsuwan et al., 2010; Vegendla, et al, 2011, Lopes et al., 2012); pero a la fecha no se ha realizado un estudio donde se propongan dos salidas colocadas asimétricamente como el estudio mostrado en este trabajo.

En este trabajo, se incluye como modelo de arrastre el EMMS (Energy Minimization Multi-Scale), el cual fue originalmente desarrollado por Yang y colaboradores $(2003,2004)$ como parte del desarrollo de la teoría MultiEscala (Li et al., 2013); la cual ha sido utilizada por varios investigadores prediciendo adecuadamente el comportamiento del flujo gas-sólidos (Wang et al., 2012; Hong et at., 2012; Wang et al., 2013; Zhou et al., 2014; Zhang et al., 2014; Liu et al., 2014).

\section{DESCRIPCIÓN DEL SISTEMA Y DOMINIO COMPUTACIONAL}

El riser que se toma como base para este estudio pertenece al sistema de refinación de México, y el modelo numérico que se propone retoma en forma general sus dimensiones y características. Se utiliza una geometría en 2D ya que según ha sido establecido por algunos autores (Periano et al., 2001; Li et al., 2014) un modelo en 2D es válido para realizar un análisis preliminar de las variables analizadas. El riser propuesto tiene un diámetro constante de $1.2 \mathrm{~m}$ y una altura de $40.0 \mathrm{~m}$, dimensiones similares al riser industrial, excepto por el detalle que el diámetro de este último varia a lo largo del riser. El vapor de fluidización se alimenta por el fondo del riser, mientras que los sólidos semi-fluidizados entran al riser a través de una tubería lateral a $45^{\circ}$ respecto a la vertical, y al entrar en contacto con la corriente de vapor, se produce la fluidización completa de los sólidos, que posteriormente incrementan su velocidad al ponerse en contacto con la corriente de gasóleo atomizado y vaporizado con la que se encuentre más arriba en el riser (ver Fig. 1). La fase granular se considera mono-dispersa con sólidos esféricos de $70 \mu \mathrm{m}$ de diámetro, y una densidad de $1,500 \mathrm{~kg} / \mathrm{m}^{3}$, que corresponde a la clasificación Geldart A (Geldart, 1973).

\section{MODELO}

El modelo que se resuelve en este trabajo para simular el comportamiento hidrodinámico y térmico del flujo gassólidos dentro del riser, está formado por las ecuaciones de balance de masa, momentum y energía; así como un conjunto de ecuaciones de cierre o constitutivas, que se utilizan para calcular la interacción entre fases, de las fases con las paredes, y las propiedades de la fase sólida que surgen de la Teoría Cinética del Flujo Granular (KTGF) (Gidaspow, 1993). Por otra parte, como modelo de arrastre se utiliza el denominado Energy Minimization Multi-Scale (EMMS) (Yang et al. 2003, 2004). 


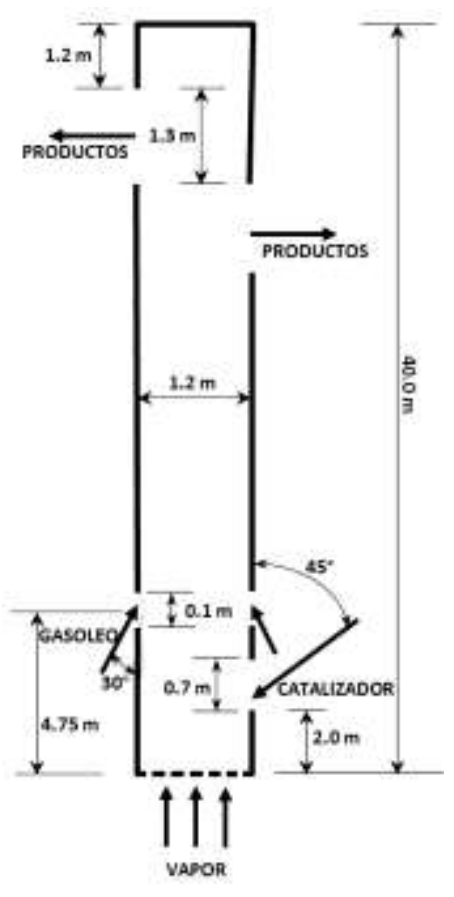

(a)

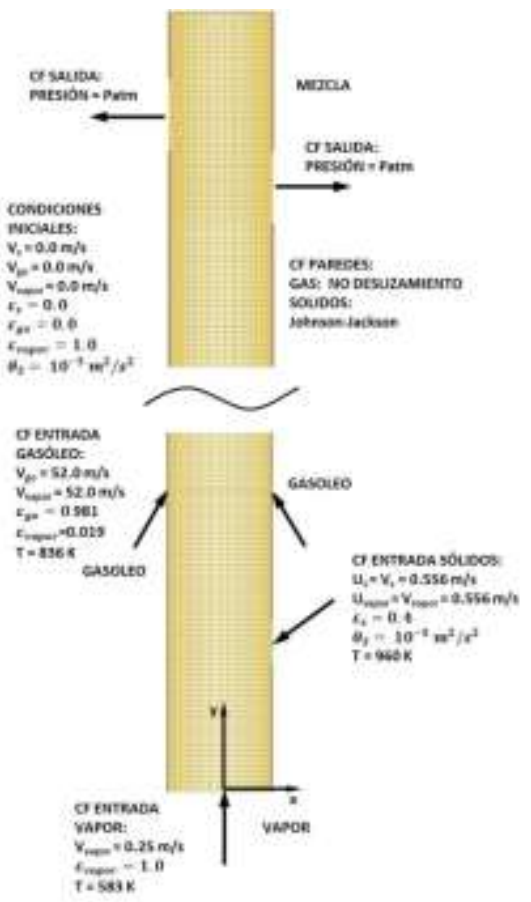

(b)

Fig. 1: Esquema y dominio computacional del riser

A continuación se muestran las ecuaciones del modelo utilizado en este trabajo. Este conjunto de ecuaciones se resuelve utilizando en programa ANSYS Fluent®, versión 14.5. El modelo EMMS no está incluido en Fluent, por lo que se desarrolla una función de usuario (UDF) (Ansys 2012a, 2012b) en lenguaje $C$ que se compila e incluye dentro del solucionador.

\section{Balance de la materia}

Fase gas

$$
\frac{\partial}{\partial t}\left(\varepsilon_{g} \rho_{g}\right)+\nabla \cdot\left(\varepsilon_{g} \rho_{g} v_{g}\right)=0
$$

Fase sólida

$$
\frac{\partial}{\partial t}\left(\varepsilon_{s} \rho_{s}\right)+\nabla \cdot\left(\varepsilon_{s} \rho_{s} v_{s}\right)=0
$$

Balance de momentum

Fase gas

$$
\frac{\partial}{\partial \mathrm{t}}\left(\varepsilon_{\mathrm{g}} \rho_{\mathrm{g}} \mathrm{v}_{\mathrm{g}}\right)+\nabla \cdot\left(\varepsilon_{\mathrm{g}} \rho_{\mathrm{g}} \mathrm{v}_{\mathrm{g}} \mathrm{v}_{\mathrm{g}}\right)=-\varepsilon_{\mathrm{g}} \nabla p+\nabla \cdot \tau_{\mathrm{g}}+\varepsilon_{\mathrm{g}} \rho_{\mathrm{g}} \mathrm{g}-\beta_{\mathrm{gs}}\left(\mathrm{v}_{\mathrm{g}}-\mathrm{v}_{\mathrm{s}}\right)
$$

Fase sólida

$$
\frac{\partial}{\partial \mathrm{t}}\left(\varepsilon_{\mathrm{s}} \rho_{\mathrm{s}} \mathrm{v}_{\mathrm{s}}\right)+\nabla \cdot\left(\varepsilon_{\mathrm{s}} \rho_{\mathrm{s}} \mathrm{v}_{\mathrm{s}} \mathrm{v}_{\mathrm{s}}\right)=-\varepsilon_{\mathrm{s}} \nabla p+\nabla \cdot \tau_{\mathrm{s}}-\Delta p_{\mathrm{s}}+\varepsilon_{\mathrm{s}} \rho_{\mathrm{s}} \mathrm{g}+\beta_{\mathrm{gs}}\left(\mathrm{v}_{\mathrm{g}}-\mathrm{v}_{\mathrm{s}}\right)
$$

Balance de energía

\section{Fase gas}

$$
\frac{\partial}{\partial \mathrm{t}}\left(\varepsilon_{\mathrm{g}} \rho_{\mathrm{g}} \mathrm{h}_{\mathrm{g}}\right)+\nabla \cdot\left(\varepsilon_{\mathrm{g}} \rho_{\mathrm{g}} \mathrm{v}_{\mathrm{g}} \mathrm{h}_{\mathrm{g}}\right)=-\varepsilon_{\mathrm{g}} \frac{\partial p_{g}}{\partial \mathrm{t}}+\tau_{\mathrm{g}}: \Delta \cdot v_{g}+\mathrm{S}_{\mathrm{g}}+\mathrm{Q}_{\mathrm{sg}}
$$


Siendo $h_{g}=\int C_{p, g} d T_{g}$

Fase sólida

$\frac{\partial}{\partial \mathrm{t}}\left(\varepsilon_{\mathrm{s}} \rho_{\mathrm{s}} \mathrm{h}_{\mathrm{s}}\right)+\nabla \cdot\left(\varepsilon_{\mathrm{s}} \rho_{\mathrm{s}} \mathrm{v}_{\mathrm{s}} \mathrm{h}_{\mathrm{s}}\right)=-\varepsilon_{s} \frac{\partial p_{g}}{\partial \mathrm{t}}+\tau_{\mathrm{s}}: \Delta \cdot v_{s}+\mathrm{S}_{\mathrm{g}}+\mathrm{Q}_{\mathrm{gs}}$

Siendo $h_{s}=\int C_{p, s} d T_{s}$

Balance de la energía fluctuante de la fase sólida

$$
\frac{3}{2}\left[\frac{\partial}{\partial \mathrm{t}}\left(\varepsilon_{\mathrm{s}} \rho_{\mathrm{s}} \theta\right)+\nabla \cdot\left(\varepsilon_{\mathrm{s}} \rho_{\mathrm{s}} \theta\right) \mathrm{v}_{\mathrm{s}}\right]=\left(-\nabla \mathrm{p}_{\mathrm{s}} \overline{\mathrm{I}}+\tau_{\mathrm{s}}\right): \nabla \mathrm{v}_{\mathrm{s}}+\nabla \cdot\left(\kappa_{\mathrm{s}} \nabla \theta\right)-\gamma_{\mathrm{s}}+\phi_{\mathrm{s}}
$$

Ecuaciones constitutivas o de cierre

Esfuerzo cortante de la fase gas

$$
\tau_{g}=\varepsilon_{g} \mu_{g}\left[\nabla v_{g}+\left(\nabla v_{g}\right)^{T}\right]-\frac{2}{3} \varepsilon_{g} \mu_{g}\left(\nabla \cdot v_{g}\right) \cdot I
$$

Esfuerzo cortante de la fase sólida

$$
\tau_{s}=\varepsilon_{s} \mu_{s}\left[\nabla v_{s}+\left(\nabla v_{s}\right)^{T}\right]+\varepsilon_{s}\left(\xi_{s}-\frac{2}{3} \mu_{s}\right) \nabla v_{s} \cdot I
$$

Disipación por colisión de la energía fluctuante de la fase sólida

$$
\gamma_{s}=3\left(1-e^{2}\right) \varepsilon_{s}^{2} \rho_{s} g_{0} \theta\left(\frac{4}{d} \sqrt{\frac{\theta}{\pi}}\right)
$$

Conductividad de la energía fluctuante de la fase sólida

$$
\kappa_{s}=\frac{150 \rho_{s} d_{p} \sqrt{\theta \pi}}{384(1+e) \mathrm{g}_{0}}\left[1+\frac{6}{5} \varepsilon_{s} \mathrm{~g}_{0}(1+e)\right]^{2}+2 \rho_{s} \varepsilon_{s}^{2} d_{p}(1+e) \mathrm{g}_{0} \sqrt{\frac{\theta}{\pi}}
$$

Función de distribución radial

$$
\mathrm{g}_{0}=\left[1-\left(\frac{\varepsilon_{s}}{\varepsilon_{\mathrm{s}, \max }}\right)^{1 / 3}\right]^{-1}
$$

Presión de la fase sólida

$$
p_{s}=\varepsilon_{s} \rho_{s} \theta\left[1+2 \mathrm{~g}_{0} \varepsilon_{s}(1+e)\right]
$$

Viscosidad de esfuerzo cortante de la fase sólida

$$
\mu_{S}=\frac{4}{5} \varepsilon_{S} \rho_{S} d_{p} \mathrm{~g}_{0}(1+e) \sqrt{\frac{\theta}{\pi}}+\frac{10 \rho_{S} d_{p} \sqrt{\pi \theta}}{96(1+e) \mathrm{g}_{0} \varepsilon_{S}}\left[1+\frac{4}{5} \mathrm{~g}_{0} \varepsilon_{S}(1+e)\right]^{2}
$$

Viscosidad promedio de la fase sólida 


$$
\xi_{s}=\frac{4}{3} \varepsilon_{s} \rho_{s} d_{p} g_{0}(1+e) \sqrt{\frac{\theta}{\pi}}
$$

Coeficiente de intercambio de momentum interfacial gas-sólidos: Modelo de arrastre EMMS

Cuando $\varepsilon_{g}<0.74$ :

$$
\beta_{\mathrm{gs}}=150 \frac{\left(1-\varepsilon_{\mathrm{g}}\right)^{2} \mu_{\mathrm{g}}}{\varepsilon_{g} d_{p}^{2}}+1.75 \frac{\left(1-\varepsilon_{g}\right) \rho_{\mathrm{g}}\left|\mathrm{v}_{\mathrm{s}}-\mathrm{v}_{\mathrm{g}}\right|}{d_{p}}
$$

Cuando $\varepsilon_{g} \geq 0.74$

$$
\beta_{\mathrm{gs}}=\frac{3}{4} \frac{\left(1-\varepsilon_{\mathrm{g}}\right) \varepsilon_{\mathrm{g}} \rho_{\mathrm{g}}}{\mathrm{d}_{\mathrm{s}}} \mathrm{C}_{\mathrm{DO}}\left|\mathrm{v}_{\mathrm{s}}-\mathrm{v}_{\mathrm{g}}\right| \omega(\varepsilon)
$$

Siendo:

$$
\begin{aligned}
& C_{D O}=\frac{24}{R e_{k}}\left(1+0.15 R e_{k}^{0.687}\right), \quad R e_{k}<1000 \\
& C_{D O}=0.44, \quad R e_{k} \geq 1000
\end{aligned}
$$

Donde:

$$
R e_{k}=\frac{\rho_{g} \varepsilon_{g}\left|v_{g}-v_{s}\right| d_{p}}{\mu_{g}}
$$

Cuando, $0.74 \leq \varepsilon_{g} \leq 0.82$ :

$$
\omega(\varepsilon)=-0.5760+\frac{0.0214}{4\left(\varepsilon_{g} 0.7463\right)^{2}+0.0044}
$$

Cuando, $0.82 \leq \varepsilon_{g} \leq 0.97$ :

$$
\omega(\varepsilon)=-0.0101+\frac{0.0038}{4\left(\varepsilon_{g} 0.7739\right)^{2}+0.0040}
$$

Cuando, $\varepsilon_{g}>0.97$ :

$$
\omega(\varepsilon)=-31.8295+32.8295 \varepsilon_{g}
$$

\section{ESTRATEGÍA DE SOLUCIÓN}

La solución del modelo CFD aplicado al flujo gas-sólidos se puede realizar a través de dos aproximaciones: Lagrangiana o Euleriana. En la aproximación Lagrangiana las fases de tratan como totalmente independientes, y el seguimiento de la fase granular se realiza siguiendo a cada una de las partículas que forma esta fase, lo que la hace poco práctica por requerir una gran cantidad de recursos computacionales. Por otra parte en la aproximación Euleriana, se considera que las fases son mutuamente inter-penetrantes y se modelan a través de la Teoría de los Dos Fluidos (TFM). En el presente trabajo el modelo propuesto se resuelve a través de una aproximación Euleriana, resolviendo las ecuaciones de balance de cada fase en forma simultánea, por la técnica de los volúmenes finitos. El sistema se resuelve en forma transitoria con un tamaño de paso de $1 \mathrm{~ms}$. Para la discretización espacial se utilizan los métodos listados en la Tabla 1; mientras que la discretización temporal se aproxima a través de un esquema implícito de segundo orden (Ansys, 2012a, 2012b). 
La simulación inicialmente se corre durante $10 \mathrm{~s}$ alimentando exclusivamente vapor de agua y posteriormente se inicia la alimentación del catalizador sólido y del gasóleo para emular la forma en que se realiza el arranque de una planta real. Posteriormente se permite que el sistema alcance el estado estacionario, lo cual se confirma monitoreando las variables analizadas cada $5 \mathrm{~s}$, hasta que se detecta que no existe cambio en sus valores, lo cual ocurre a los $20 \mathrm{~s}$. A partir de este tiempo y hasta el tiempo final de la simulación a los $50 \mathrm{~s}$ se toman los promedios de las variables analizadas.

Tabla 1. Esquema numérico para la discretización espacial. ANSYS (2012a, 2012b)

\begin{tabular}{|l|l|}
\hline Propiedad & Esquema numérico \\
\hline Gradiente & Mínimos cuadrados \\
\hline Momentum & Power law \\
\hline Fracción Volumétrica & Upwind primer orden \\
\hline Temperatura Granular & Power law \\
\hline Energía & Power law \\
\hline
\end{tabular}

Adicionalmente se definen condiciones iniciales y de frontera para resolver el conjunto de ecuaciones diferenciales parciales. Como condición inicial se establece que el riser sólo está lleno de vapor, y que la temperatura granular de los sólidos tiene un valor de $10^{-5} \mathrm{~m}^{2} / \mathrm{s}^{2}$. Como condiciones de frontera se definen las velocidades, concentraciones y temperaturas de las corrientes de entrada (ver Figura 1a). Adicionalmente se definen condiciones de frontera de los sólidos en las paredes del riser (Johnson y Jackson, 1987).

\section{RESULTADOS Y DISCUSIÓN}

Las variables obtenidas directamente de la simulación son la fracción volumétrica, la velocidad, y la temperatura de los sólidos. A partir de las dos primeras variables se calcula: la densidad, y el flux másico de los sólidos, siendo estas últimas variables las que comúnmente se reportan para analizar los lechos fluidizados del Proceso FCC. En la Figura 2 se puede ver la densidad de sólidos a diferentes alturas del riser para el caso analizado en este trabajo, y en la Figura 3 se muestra la misma variable para en caso de salidas simétricas analizadas en un trabajo previo (Paz-Paredes, 2016).

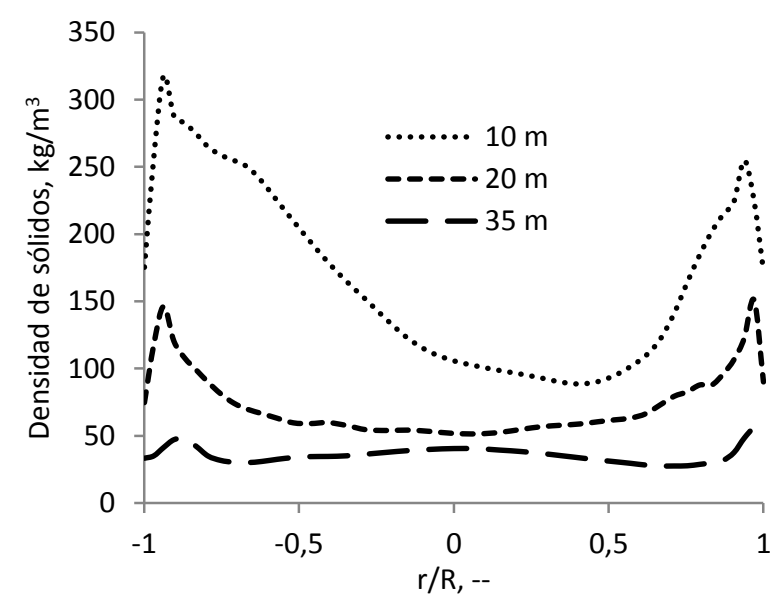

Fig. 2: Densidad de sólidos caso salidas asimétricas, $\mathrm{kg} / \mathrm{m}^{3}$

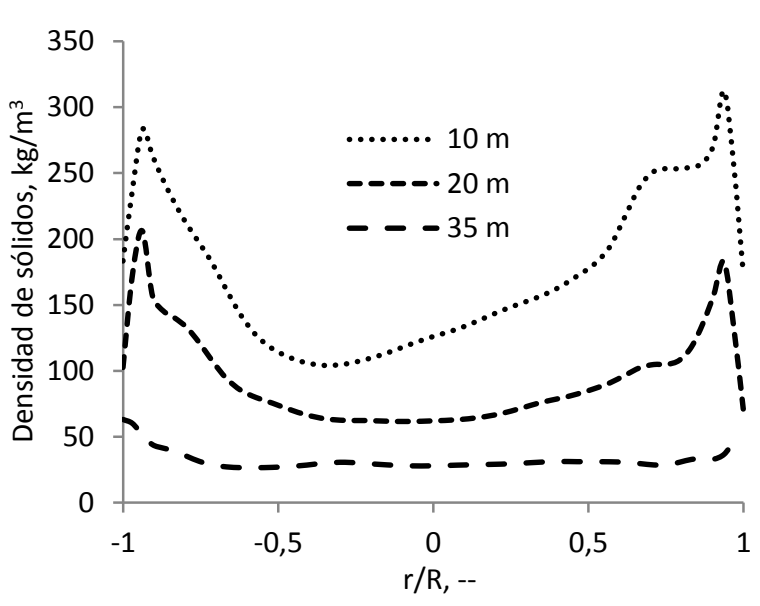

Fig. 3: Densidad de sólidos salidas simétricas, $\mathrm{kg} / \mathrm{m}^{3}$

Se observa que en la parte media del riser la concentración de lo sólidos cerca de las paredes es mayor para el caso de salidas simétricas, mientras que en la parte baja y alta las concentraciones son similares. Se puede ver que el hecho de tener una salida colocada más abajo como en el caso asimétrico, favorece la concentración de sólidos en el lado contrario del riser, lo cual podría ser usado en el caso de que se requiera tal concentración para casos específicos. De igual manera que para la densidad de sólidos, en la Figura 4 y 5 se muestran las velocidades de los sólidos a varias alturas de riser, para el caso de salidas asimétricas y simétricas, respectivamente. Para el caso de las salidas asimétricas se observa que los perfiles de velocidad tienen tendencia hacía donde se encuentra la salida colocada más abajo en el riser, mientras que para las salidas simétricas en la parte baja y alta la tendencia del perfil de velocidad es hacia el lado contrario de donde entran los sólidos, y para la parte media el perfil es casi simétrico. 


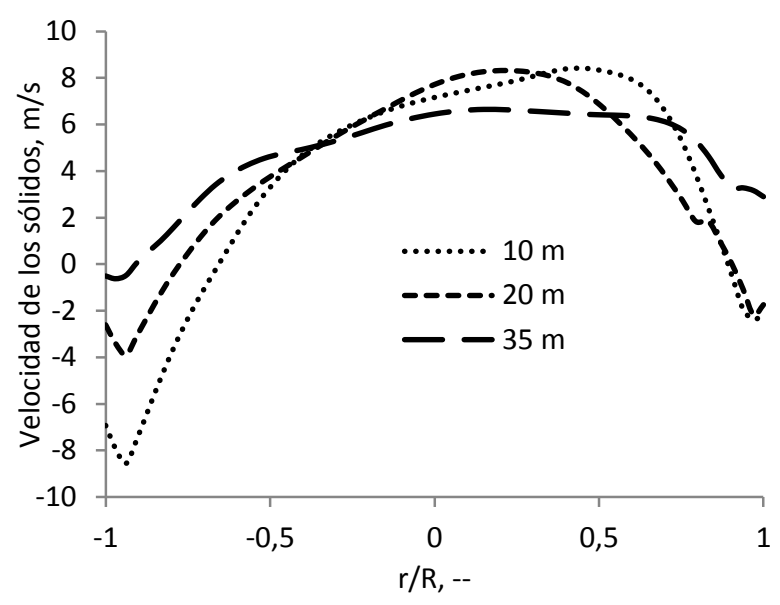

Fig. 4: Velocidad axial de los sólidos caso salidas asimétricas, $\mathrm{m} / \mathrm{s}$

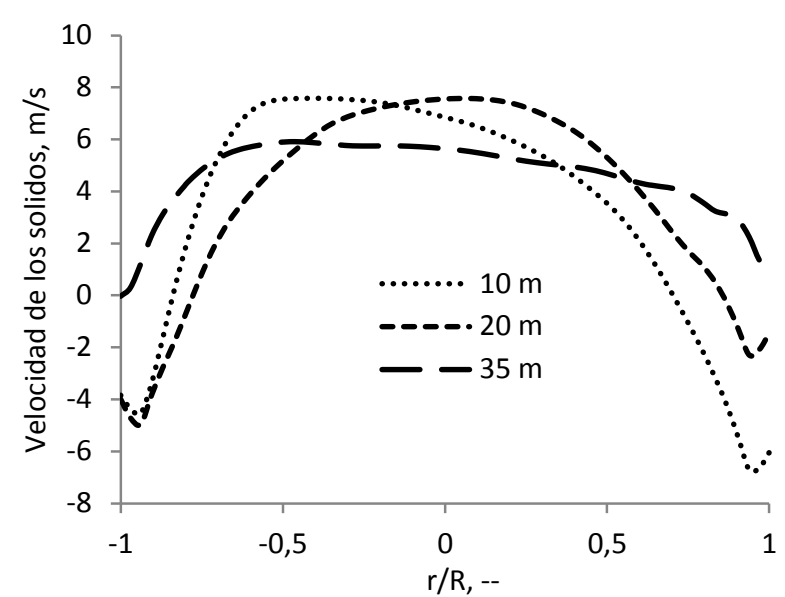

Fig. 5: Velocidad axial de los sólidos caso salidas simétricas, $\mathrm{m} / \mathrm{s}$

Por otra parte, en ambos casos se presenta el flujo de sólidos moviéndose en forma descendente en la parte baja y media de ambos casos; sin embargo las velocidades de descenso en la parte baja son mayores en el lado contrario de la salida asimétrica, mientras que para el caso de las salidas simétricas, las velocidades de descenso se presentan del mismo lado donde entran los sólidos. En la parte media del riser las velocidades de descenso de los sólidos tienen aproximadamente el mismo comportamiento, mientras que en la parte alta del riser en ambos casos los sólidos se mueven sólo en dirección ascendente, esto se puede explicar por la considerable disminución de la concentración de sólidos, lo que aumenta su velocidad y ya no se presenta el movimiento de los sólidos en dirección descendente.

En la Figura 6 se muestra el flux másico de sólidos -flujo másico de sólidos por unidad de tiempo y de área-, y en la Figura 7 se muestra el comportamiento de la temperatura promedio de los sólidos, ambas variables a diferentes alturas del riser para el caso de salidas asimétricas. En la parte baja del riser el flux másico de sólidos muestra una gran cantidad de sólidos moviéndose en forma descendente en el lado contrario a donde se encuentra la salida colocada a menor altura; conforme aumenta la altura, la concentración de sólidos, y la velocidad descendente disminuyen en la parte media y se mantiene un poco menor en la parte alta. La temperatura promedio de los sólidos es estabiliza en aproximadamente $920 \mathrm{~K}$, excepto en la parte baja de riser donde se observa una menor temperatura cerca de las paredes del riser, siendo más marcado este descenso en el lado donde está colocada la salida más baja. Es claro que el flujo preferencial hacia la salida a menor altura afecta la temperatura en la parte baja del riser.

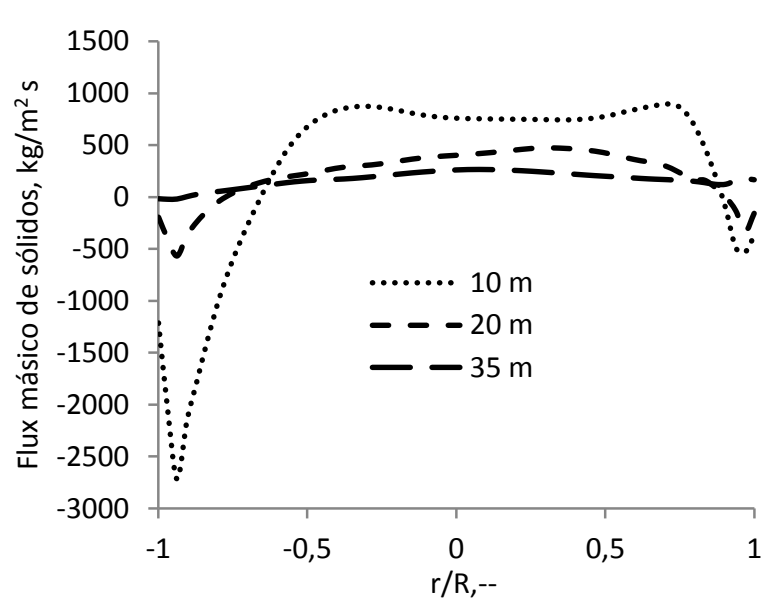

Fig. 6: Flujo másico de sólidos caso salidas asimétricas, $\mathrm{kg} / \mathrm{m}^{2} \mathrm{~s}$

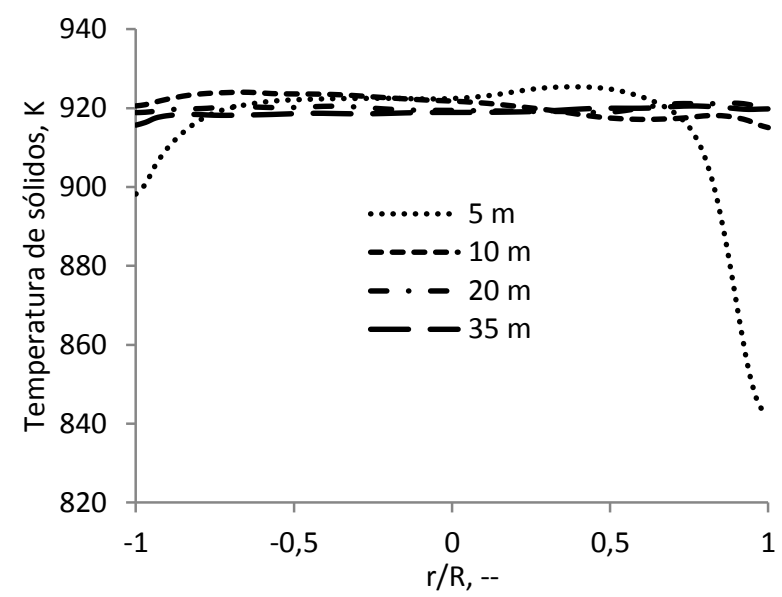

Fig. 7: Temperatura de sólidos caso salidas asimétricas, $\mathrm{K}$

En la Figura 8 se muestra el contorno de la fracción volumétrica de los sólidos tanto los valores promedio entre 20 y $50 \mathrm{~s}$, y los valores instantáneos al tiempo final de $50 \mathrm{~s}$; mientras que en la Figura 9 de muestran los valores de la velocidad de sólidos a las mismas condiciones. Los valores promedio emulan los valores que se toman de los instrumentos en las corridas experimentales o industriales, mientras que los valores instantáneos proporcionan información del comportamiento de las variables en un tiempo específico. Así pues, en los contornos promedio se puede ver la predicción de las zonas concentradas y diluidas de sólidos en la parte baja y alta del riser, respectivamente; mientras que la fracción volumétrica instantánea permite ver zonas de alta concentración de 
sólidos inmersas en zonas de menor concentración, lo que significa que el modelo propuesto predice la formación de grupos de partículas (clusters). En el contorno de velocidad instantánea se puede observar el comportamiento oscilatorio del flujo de los sólidos.

En la Figura 10 se presenta el contorno de temperatura promedio de los sólidos, donde claramente se pueden diferenciar tres zonas: en la parte baja se presenta la temperatura más elevada debido que ahí se alimenta el catalizador caliente, enseguida casi la totalidad del riser a la temperatura promedio de aproximadamente $920 \mathrm{~K}$, lo cual tampien se puede ver en la Figura 7 anterior; finalmente la zona alta del riser donde se presentan las temperaturas más bajas debido a la presencia de una zona estancada de sólidos, lo cual se confirma en las Figuras 8 y 9 donde también se presenta dicha zona.

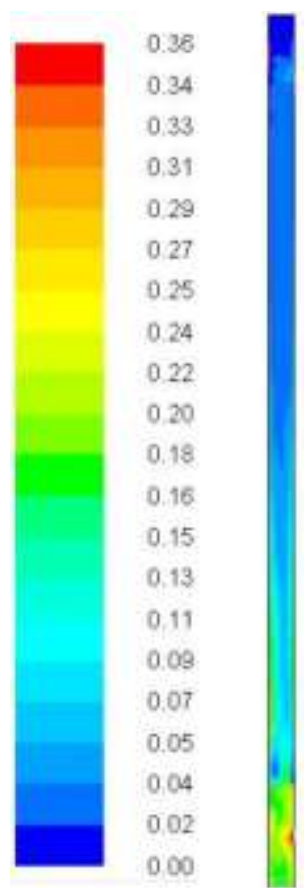

Promedio

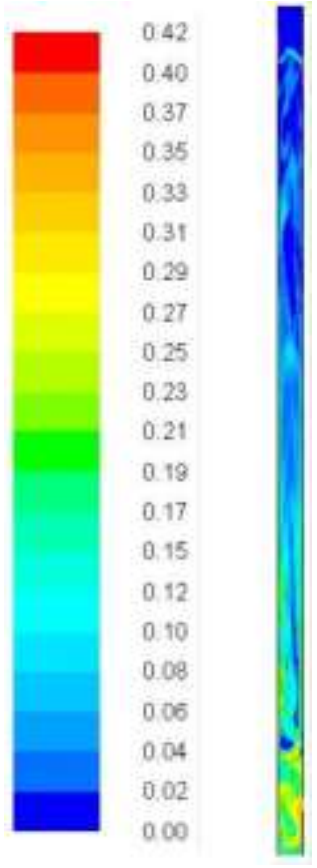

Instantánea a 50 s

Fig. 8: Fracción volumétrica de sólidos caso salidas asimétricas
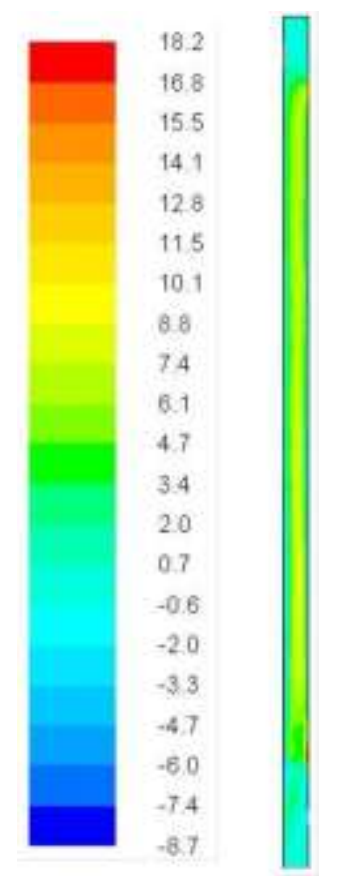

Promedio

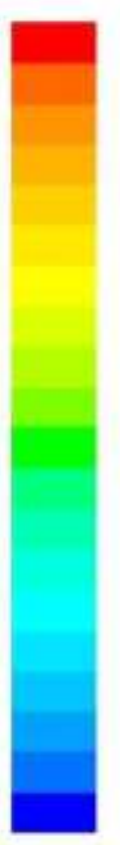

19.6
18.1
16.6
15.0
13.5
12.0
10.4
8.9
7.4
5.8
4.3
2.8
1.2
-0.3
-1.8
-3.4
-4.9
-6.4
-7.9
-9.5
-11.0

Instantánea a $50 \mathrm{~s}$

Fig. 9: Velocidad de los sólidos caso salidas asimétricas, $\mathrm{m} / \mathrm{s}$

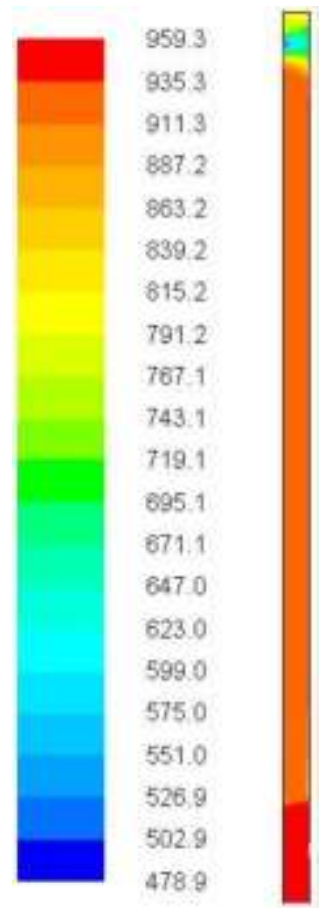

Fig. 10: Temperatura promedio de los sólidos, $\mathrm{K}$ 


\section{CONCLUSIONES}

De acuerdo a los resultados obtenidos en esta investigación, se pueden establecer las siguientes conclusiones: 1) El modelo CFD propuesto incluyendo el modelo de arrastre EMMS, predice adecuadamente las diferentes zonas de concentración de sólidos, así como la formación de grupos de partículas y los patrones de flujo con sólidos fluyendo en dirección descendente cerca de las paredes, que concuerdan con trabajos previos realizados. 2) La dos configuraciones de salidas simétricas y asimétricas producen patrones de flujo anular, sin embargo en el caso de salidas asimétricas se producen un flujo preferencial hacia la salida a menor altura como era esperado, evidenciado por mayores velocidades y menores concentraciones de sólidos en esa zona. 3) Para las condiciones de operación del riser, y tipo de sólidos, la densidad de sólidos más homogénea se presenta en la parte alta del riser, donde se tiene una densidad de sólidos de aproximadamente $50 \mathrm{~kg} / \mathrm{m}^{3}$, y una velocidad entre 3 y $6 \mathrm{~m} / \mathrm{s}$. 4) Esta condición de concentración de sólidos homogénea es lo que se busca para favorecer el rendimiento de las reacciones químicas del proceso FCC, lo cual se comprobará en un trabajo futuro cuando se incluyan al modelo de este trabajo, los modelos de transferencia de masa y cinéticos.

\section{AGRADECIMIENTOS}

Se agradece el apoyo del Instituto Mexicano del Petróleo al otorgar una beca a través de una licencia con goce de sueldo, para que el autor principal realice estudios de doctorado, dentro de los cuales se desarrolla la presente investigación.

\section{REFERENCIAS}

Ansys, Ansys Fluent User's Guide, Release 14.5, Canonsburg, PA (2012a)

Ansys, Ansys Fluent Tutorial's Guide, Release 14.5, Canonsburg, PA (2012b)

Cabezas-Gómez, L., y F.E., Milioli, Numerical Study on the Influence of various Physical Parameters Over the Gas-Solid Two-Phase Flow in the 2D Riser of a Circulating Fluidized Bed, doi:10.1016/S0032-5910(03)000718, Powder Technol. (en linea), 132(2-3), 216-225 (2003)

Chalermsinsuwan, B., P. Kuchonthara, y P. Piumsomboom, CFD Modeling of Tapered Circulating Fluidized Bed Reactor Risers: Hydrodynamic description and Chemical Reaction Responses, doi:10.1016/j.cep.2010.08.016, Chem. Eng. Process. (en linea), 49(11), 1144-1160 (2010)

Geldart, D., Types of gas fluidization, doi: 10.1016/0032-5910(73)80037-3, Powder Technol. (en linea), 7(5), 285-292 (1973)

Gidaspow, D, Multiphase Flow and Fluidization-Continuum and Kinetic Theory Descriptions, Academic Press, San Diego, USA (1994)

Hamzehei, M., y H. Rahimzadeh, Numerical and Experimental Investigation of a Fluidized Bed Chamber Hydrodynamics with Heat Transfer, Korean J. Chem. Eng., 27(1), 355-363 (2010)

Huilin, L., Z. Yunhua, S. Zhiheng S., J. Ding J. y J. Jiying, Numerical Simulations of Gas-Solid Flow in Tappered Risers, doi:10.1016/0032-5910(73)80037-3, Powder Technol., 169(2), 89-98 (2006)

Hong, K., W. Wang, Q. Zhou, J. Wang y J. Li, An EMMS Multi-Fluid Model (EFM) for Heterogeneous GasSolid Riser Flows: Part I. Formulation of Structure-Dependent Conservation Equations, doi:10.1016/j.ces.2012.03.022, Chem. Eng. Sci. (en linea), 75, 376-389 (2012)

Johnson, P.C., y R. Jackson, Frictional-Collisional Constitutive Relations for Granular Materials, with Application to Plane Shearing, doi: http://dx.doi.org/10.1017/S0022112087000570, J. Fluid Mech. (en linea), 176, 67-93 (1987)

Li, J., W. Ge, W. Wang, N. Yang, X. Liu, L. Wang, X. He, X. Wang, J. Wang y M. Kwauk, From Multiscale Modeling to Meso-Science. A Chemical Perspective. Principles, Modeling, Simulation, and Application, Springer-Verlag Berlin Heidelberg (2013)

Li, T., S. Pannala, y M. Shahnam, CFD simulations of circulating fluidized bed risers, part II: evaluation of differences between 2D and 3D simulations, doi:10.1016/j.powtec.2014.01.022, Powder Technol. (en linea), 254, 115-124 (2014) 
Liu, X., Y. Jiang, C. Liu, W. Wang y J. Li, Hydrodynamic Modeling of Gas-solid Bubbling Fluidization based on Energy Multiscale (EMMS) Theory, doi 10.1021/ie4029335. Ind. Eng. Chem. Res. (en linea), 53(7), 2800-2810 (2014)

Lopes, G.C., L.M. Rosa, M. Mori, J.R. Nunhez y W.P. Martignioni CFD Study of Industrial FCC Risers: The Effect of Outlet Configurations on Hydrodynamics and Reactions, Int. J. Chem. Eng., 2012, Article ID 193639 (2012)

Neri, A., y D. Gidaspow, Riser Hydrodynamics: Simulation Using Kinetic Theory, doi: 10.1002/aic.690460108, AIChE J. (en linea), 46(1), 52-66 (2000)

Pärssinen, J.H., y J. X. Zhu, Particle Velocity and Flow development in a Long and High Flux Circulating Fluized Bed Riser, Chem. Eng. Sci., 56, 5295-5303 (2001)

Paz-Paredes, I, Tesis Doctoral. ESIME. Instituto Politécnico Nacional. México. (2016)

Peirano, E., V. Delloume y B. Lecker, Two or three-dimensional simulations of turbulent gas-solid flows applied to fluidization, doi:10.1016/S0009-2509(01)00141-5, Chem. Eng. Sci. (en linea), 56(16), 4787-4799 (2001)

Reddy-Kerri, S.B. y T.M. Knowlton, A comparison of Annulus Solids Flow Direction and radial Solids Mass Flux Profiles at Low and High Mass Fluxes in a Riser, Circulating Fluidized Bed Technology, Werther J. Ed., Frankfurt: DECHEMA, 71-76 (1999)

Sadeghbeigi, R. Fluid Catalytic Cracking Handbook, Gulf Publishing Company, Houston, USA (1995)

Vegendla, S.N.P., G.J. Hyenderickx y G.B. Marin, Comparison of Eulerian-Lagrangian and Eulerian-Eulerian Method for Dilute Gas-Solid Flow with Side Inlet, doi:10.1016/j.compchemeng.2010.09.001, Comp. Chem Eng. (en linea), 35(7), 1192-1199 (2011)

Wang, J., Q. Zhou, W. Wang y J. Li, An EMMS Multi-Fluid Model (EFM) for Heterogeneous Gas-Solid Riser Flows: Part II. An Alternative Formulation from Dominant Mechanisms, doi:10.1016/j.ces.2012.03.021, Chem. Eng. Sci. (en linea), 75, 349-358 (2012)

Wang, S., H. Lu, D. Li, y Y. Tang, Simulation of the Chemical Looping Reforming Process in the Fuel Reactor with Bubble-Based Energy Minimization Multiscale Model, doi: 10.1021/ef401101p, Energy Fuels (en linea), 27(8), 5008-5015 (2013)

Wen, C., y Y. Yu, Mechanics of fluidization, Chem. Eng. Prog. Symp. Ser., 62, 100-111 (1966)

Yang, N., W. Wang, W. Ge y J. Li, Choosing Structure-Dependent Drag Coefficient In Modeling Gas-Solid Two-Phase Flow, doi:10.1016/S1672-2515(07)60098-0, China Particuology (en linea), 1(1), 38-41 (2003)

Yang, N., W. Wang, W. Ge, L. Wang y J. Li, Simulation of Heterogeneous Structure in a Circulating FluidizedBed Riser by Combining the Two-Fluid Model with the EMMS Approach, doi: 10.1021/ie049773c, Ind. Eng. Chem. Res. (en linea), 43(18), 5548-5561 (2004)

Zhang, L., X. Qiu, L. Wang y J. Li, A Stability Condition for Turbulent Model: From EMMS to EMMS-Based Turbulence Model, doi:10.1016/j.partic.2014.02.003, Particuology (en linea), 16, 142-154 (2014)

Zhou, Q., J. Wang y J. Li, Three-dimensional Simulations of Dense Suspension Upflow Regime in High-Density CFD Risers with EMMS-Based Two-Fluid Model, doi:10.1016/j.ces.2013.12.020, Chem. Eng. Sci. (en linea), 107, 206-217 (2014) 